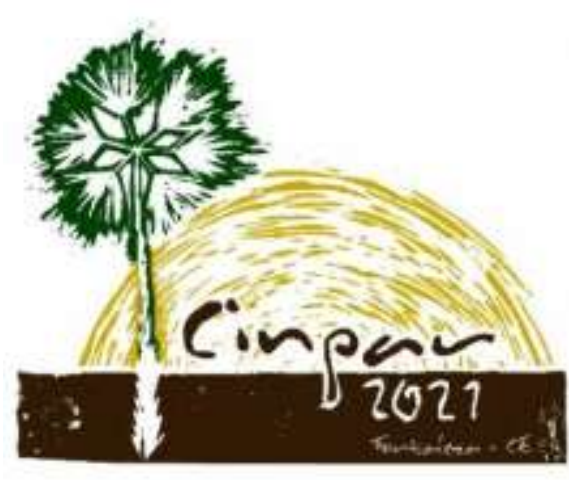

XVII Congresso Internacional sobre Patologia e Reabilitação das Construções

XVII Congreso Internacional sobre Patología y Rehabilitación de las Construcciones

XVII International Conference on Pathology and Constructions Rehabilitation

FORTALEZA (Brasil), 3 a 5 de junho de 2021 https://doi.org/10.4322/CINPAR.2021.157

\title{
Inspeção Predial em Empreendimentos Rurais Aplicabilidade da NBR 16747
}

\section{Building Inspection in Rural Enterprises Applicability of NBR 16747}

\author{
Camilla Cristina Cunha MENEZES ${ }^{1}$, Marcos de Paulo RAMOS², Thiago Pena BORTONE ${ }^{3}$, Rachel Jardim \\ MARTINI ${ }^{4}$
}

\author{
${ }^{1}$ CEFET/MG, Curvelo, Brasil, millacunha12@gmail.com \\ ${ }^{2}$ CEFET/MG, Curvelo, Brasil, marcos.ramos@cefetmg.br \\ ${ }^{3}$ CEFET-MG, Curvelo, Brasil, thiago.bortone@cefetmg.br \\ ${ }^{4}$ CEFET-MG, Curvelo, Brasil, martini@cefetmg.br
}

\begin{abstract}
Resumo: A organização orientada à exploração extrativa, agrícola ou agroindustrial para fins de beneficiamento social é denominada empreendimento rural. As construções rurais relacionadas às unidades de produção desses empreendimentos são de grande relevância e, devem atender as legislações vigentes, oferecer condições de salubridade aos colaboradores e usuários, permitindo assim a eficiência das atividades produtivas. A inspeção predial auxilia na preservação do imóvel, associada ao melhor desempenho da edificação, procurando identificar possíveis anomalias e falhas construtivas nos sistemas constituintes. Publicada em maio de 2020, pela Associação Brasileira de Normas Técnicas - ABNT, a norma NBR 16747 apresenta as orientações para a execução de inspeções prediais das edificações. O presente trabalho discute a importância da aplicação da norma técnica de Inspeção Predial (ABNT NBR 16747) em Empreendimentos Rurais. O processo de Inspeção Predial contribui na identificação precoce de manifestações patológicas nas edificações. Dessa forma, evita que anomalias; falhas construtivas; ausência de manutenções ou manutenções inadequadas; uso de materiais de qualidade inferior; não cumprimento das normas técnicas; tragam prejuízos financeiros, comprometimento da saúde e segurança da edificação e atrasos na produção. Em relação ao emprego da ABNT NBR 16747 em empreendimentos rurais, observa-se que a norma supracitada contribui fortemente na análise da situação administrativa e técnica da edificação uma vez que, em seu Anexo A, consta uma lista de itens a serem inspecionados que normalmente passam despercebidos em vistorias de manutenção. Observou-se ainda, que a etapa metodológica preconizada pela norma contribui na melhor compreensão dos problemas presentes no empreendimento rural, além cumprir com eficiência seu papel de auxiliar na gestão da edificação e na mitigação de riscos técnicos e econômicos associados à perda de desempenho.
\end{abstract}

Palavras-chave: Empreendimentos rurais, manifestações patológicas, inspeção predial.

Abstract: The organization oriented to extractive, agricultural or agro-industrial exploitation for the purpose of social improvement is called rural enterprise. Rural buildings related to the production units of these projects are of great relevance and, must comply with current legislation, offer health conditions to employees and users, allowing the efficiency of productive activities. Building inspection assists in the preservation of the property, associated with the better performance of the building, seeking to identify possible anomalies and construction failures in the constituent systems. Published in May 2020, by the Brazilian Association of Technical Standards - ABNT, NBR 16747 standard presents guidelines for carrying out buildinginspections of 
buildings. This paper discusses the importance of applying the Building Inspection technical standard (ABNT NBR 16747) in Rural Enterprises. Building Inspection processes contribute to the early identification of pathological manifestations in buildings. That way, it prevents anomalies, construction failures, absence or inadequate maintenance, use of inferior quality materials, non-compliance with technical standards, bring financial losses, compromised building health and safety and production delays. In relation to the use of ABNT NBR 16747 in rural enterprises, it is observed that the aforementioned standard strongly contributes to the analysis of the administrative and technical situation of the building since, in its Annex $A$, there is a list of items to be inspected that normally pass unnoticed in maintenance inspections. It was also observed that the methodological step recommended by the standard contributes toa better understanding of the problems present in the rural enterprise, in addition to efficiently fulfilling its auxiliary role in the management of the building and mitigation of technical and economic risks associated with the loss of performance.

Keywords: Rural enterprises, pathological manifestations, building inspection.

\section{Introdução}

As organizações rurais surgem frente a necessidade de suprir demandas do mercado consumidor, visando a diminuição dos custos de produção e aumento do faturamento. Conforme Garrido (2006), no Brasil é comum encontrar empreendimentos rurais em vários estágios de desenvolvimento, grande parte destes são embasados na agricultura tradicional com aplicação mínima de tecnologias construtivas.

Considerando essa baixa aplicação de tecnologias construtivas nos empreendimentos rurais, as Inspeções Prediais surgem como ferramenta na prevenção de acidentes, gestão da qualidade das edificações e redução de custos. Estas inspeções, segundo Verzola (2014), devem ser realizadas a partir de uma lista de verificação, de caráter preditiva, que registre o diagnóstico das falhas constatadas.

As falhas construtivas são decorrentes da ineficiência das definições de projeto, aplicação de materiais de baixa qualidade ou erros de execução, afetando a vida útil da edificação e seu desempenho no uso e operação. Outros fatores como eventos climáticos, poluição e nível de agressividade do ambiente podem acarretar na redução do prazo teórico de vida útil projetado para a edificação (ABNT, 2020).

De modo geral, a Inspeção Predial atua como um processo de constatação de falhas e manifestações patológicas, classificando-as quanto a sua gravidade e orientando o cliente quanto as ações de manutenção da edificação (ABNT, 2020). De acordo com Pujadas (2006), a Inspeção Predial deve atuar em dinâmica tríplice averiguando aspectos (i) técnicos, de (ii) uso e (iii) manutenção da edificação.

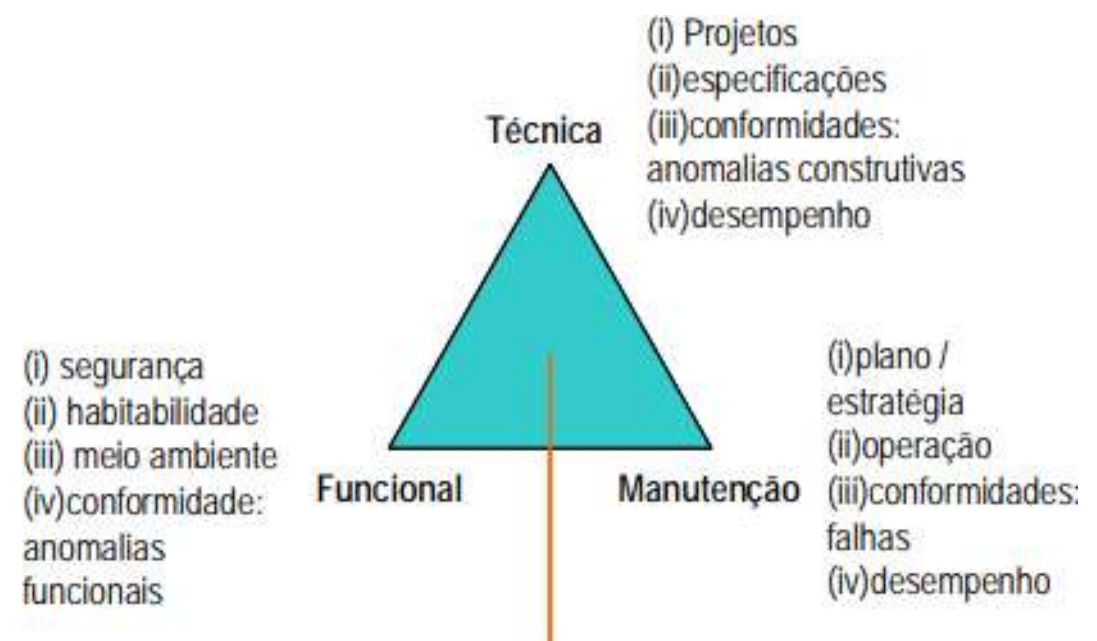

AUDITORIA TECNICA

Figura 1 - Visão tríplice da Inspeção Predial

Fonte: PUJADAS (2006). 
A NBR 16747 (ABNT, 2020), apresenta as diretrizes, conceitos e terminologias referentes ao procedimento utilizado na Inspeção Predial, itens que antes não eram normatizados pela Associação Brasileira de Normas Técnicas - ABNT, o que ocasionava discrepâncias na execução dos processos de inspeções prediais.

O objetivo deste trabalho é expor a metodologia da recente ABNT NBR 16747 (ABNT, 2020), analisando sua abrangência quanto a tipologia dos imóveis de empreendimentos rurais. A pesquisa também apresenta algumas recomendações para adaptação do check-list convencional utilizado nas vistorias, para que melhor represente a realidade da edificação rural inspecionada quanto a sua finalidade e uso.

\section{Metodologia de inspeção em edificações}

A Inspeção Predial trata-se de uma ferramenta da Engenharia que visa detectar determinado fato ou condição, mediante a verificação in loco. Na execução da inspeção de caráter diagnóstico, são identificadas eventuais anomalias e falhas construtivas. A averiguação quanto ao desempenho dos sistemas, subsistemas e componentes construtivos de uma edificação tem ganhado enfoque frente a preservação patrimonial e segurança do indivíduo.

De acordo com as necessidades de segurança, salubridade e economia são determinadas as condições mínimas de desempenho e qualidade que a edificação deve atender, verificando a adequação a utilização e funcionamento, independente das técnicas construtivas adotadas (ABNT, 2013).

\subsection{ABNT NBR 16747 - Inspeção Predial}

O exercício da verificação predial objetiva assessorar na gestão das edificações, onde é realizada uma análise sensorial das condições técnicas de uso, operação, manutenção e funcionalidade, observando a presença de problemas e agentes de degradação que acarretam a redução do desempenho do imóvel.

A metodologia da Inspeção Predial, conforme NBR 16747 (ABNT, 2020), abrange fatores técnicos de uso, operação emanutenibilidade. A norma apresenta as seguintes etapas:

a) Levantamento de dados e documentação;

b) Verificação dos dados e documentação disponibilizada;

c) Anamnese;

d) Vistoria sensorial do imóvel e das instalações;

e) Análise e classificação das anomalias levantadas;

f) Instrução quanto às ações de reparação,

g) Organização das ações de manutenção e reparo em patamares de urgência, considerando o nível de prioridade das intervenções;

h) Avaliação da manutenção;

i) Avaliação de uso da edificação;

j) Elaboração e emissão do laudo técnico.

No ramo da construção civil a anamnese trata-se do estudo prévio realizado a partir de uma entrevista com o proprietário e usuários do local, buscando coletar dados e informações que retratem o histórico do local, referente a manutenções e reformas, que possa contribuir no diagnóstico assertivo de falhas e anomalias identificadas na edificação (ABNT, 2020).

Para uma execução adequada a norma enfatiza que os profissionais habilitados a exercer a avaliação devem estar inteirados das regras vigentes. Segundo a NBR 16747 (ABNT, 2020), após a inspeção, as informações coletadas devem ser confrontadas com as exigências de projeto e execução disponibilizadas durante a fase de verificação da documentação.

Quanto a conformidade perante ao plano de manutenção, são verificados a coerência em relação as orientações técnicas, especificações do fabricante e manuais elaborados conforme ABNT NBR 14037 (ABNT, 2014)que trata das diretrizes para elaboração de manuais de uso, operação e manutenção das edificações.

Em relação ao uso, as condições podem ser regulares ou irregulares quando analisadas frente aos projetos, normas vigentes, especificações do fabricante, manuais de utilização e funcionamento. 


\subsection{Check-list de verificação}

Os empreendimentos rurais possuem em sua arquitetura características específicas de acordo com a atividade desenvolvida. O local deve manter a infraestrutura necessária para que os colaboradores desempenhem suas funções com saúde e segurança. Para que haja produção contínua, produtos e serviços de qualidade, a edificação deve manter a funcionalidade perante a eventos climáticos e metereológicos.

Segundo IBAPE (2012), o check-list de verificação deve abranger os sistemas, subsistemas, equipamentos e componentes construtivos, a serem vistoriados, sendo apropriada à complexidade do imóvel. Portanto, o check-list trata-se de um aparato que deve ser empregado nas vistorias visando a manutenção do desempenho destes empreendimentos rurais, onde verifica-se os elementos que serão vistoriados considerando o estado de preservação dos componentes. Na vistoria recomenda-se que sejam avaliadas as peças construtivas, esquadrias, sistema de vedação externos e internos, sistema de proteção contra descargas atmosféricas (SPDA), sistema de combate a incêndio, revestimentos internos e externos, cobertura, condições de uso de instalações elétricas, instalações hidráulicas e hidrosanitárias, considerando o grau de exposição ambiental, agentes externos atuantes e procedimentos operacionais.

Em relação as condições de salubridade do local, devem ser analisados os sistemas de impermeabilização, climatização, ventilação e exaustão. Estes fatores também podem interferir nas condições de trabalho dos colaboradores.

De caráter informativo, a NBR 16747 (ABNT, 2020) exibe uma tabela com os principais documentos técnicos, administrativos, de operação e manutenção que se recomenda analisar na etapa de verificação, conforme resumo apresentado no Quadro 1, porém, a norma não apresenta um check-list específico para tipologia rural, assim como os diversos modelos encontrados que possuem embasamento no ambiente urbano.

Quadro 1 - Documentação a ser analisada na etapa b)

\begin{tabular}{|c|}
\hline Documentos administrativos e técnicos \\
\hline Manual de uso, operação e manutenção da edificação \\
\hline Manual técnico de equipamentos instalados \\
\hline Alvará de funcionamento \\
\hline Auto de vistoria do corpo de bombeiros (AVCB) \\
\hline Projetos legais aprovados \\
\hline Projetos executivos \\
\hline Licenças ambientais \\
\hline Atestado de Brigada de Incêndio \\
\hline Certificado de teste dos equipamentos de combate à incêndio \\
\hline Relatório de manutenção da estação de tratamento de efluentes \\
\hline Relatório de manutenção da estação de tratamento de água \\
\hline Atestado do sistema de proteção a descarga atmosférica (SPDA) \\
\hline
\end{tabular}

Fonte: Adaptado da NBR 16747 (2020)

Ao se inspecionar as edificações de áreas rurais, o profissional pode se deparar com realidades distintas do meio urbano, além de infraestrutura precária, ineficiência dos processos e, em alguns casos, escassez de recursos básicos que são previstos por lei para a população. De acordo com Sarmento (2015), "A população rural vive em condições de desigualdade socioeconômica, motivadas por vários problemas entre eles a insuficiência de sistemas de esgoto e abastecimento de água". Como proposta de integração aos check-list's normalmente encontrados na literatura, são recomendados a seguir alguns itens de verificação de estruturas que integram alguns empreendimentos rurais. 


\subsection{Sistemas de captação de água subterrânea}

Como item essencial do check-list proposto para empreendimentos rurais, devem ser analisados os sistemas de captação de água conforme os itens exibidos no Quadro 2. Este quadro verifica as condições do reservatório frente as condições de uso e potabilidade da água, conforme a Portaria no 2914, do Ministério da Saúde, de 12 de dezembro de 2011, que apresenta os procedimentos de controle e gestão de qualidade da água para consumo, padronizando o nível de potabilidade, onde emprega-se o recurso como solução alternativa para abastecimento de água (BRASIL, 2011).

Quadro 2 - Verificação de sistemas de captação subterrânea

\begin{tabular}{|c|c|}
\hline Descrição & \\
\hline $\begin{array}{c}\text { Estado de conservação das estruturas, equipamentos e } \\
\text { dispositivos de Captação. }\end{array}$ & $\operatorname{REGULAR}($ ) IRREGULAR ( ) \\
\hline Estado de conservação das bombas e equipamentos elétricos. & $\operatorname{REGULAR~(~)~IRREGULAR~(~)~}$ \\
\hline Manutenção periódica dos dispositivos e equipamentos. & $\operatorname{REGULAR~(~)~IRREGULAR~()~}$ \\
\hline Sistema de bombeamento reserva. & $\operatorname{REGULAR~(~)~IRREGULAR~()~}$ \\
\hline
\end{tabular}

Fonte: Autores

\subsection{Esgotamento sanitário}

O saneamento básico, promove a prevenção de doenças e de proteção à saúde populacional, sendo constitucionalmente integrado ao campo da saúde e, consequentemente, ao âmbito da política social (SILVA, 2017). Observamos que, mesmo com um histórico de políticas e medidas voltadas ao desenvolvimento do saneamento básico no meio rural, muitas propriedades ainda não possuem um sistema eficiente e, em alguns casos, despejam os efluentes em locais indevidos, comprometendo mananciais superficiais e subterrâneos.

Em áreas rurais a técnica utilizada para disposição do esgoto doméstico é a fossa séptica, que são unidades que tratam o esgoto doméstico, porém não é uma opção adequada, tendo eficiência baixa e limitada. Os itens mínimos que recomendamos para análise dos sistemas de esgotamento sanitário em empreendimentos rurais estão descritos no Quadro 3.

Quadro 3 - Verificação do sistema de esgoto

\begin{tabular}{|c|l|}
\hline \multicolumn{1}{|c|}{ Descrição } & \\
\hline Caixas de gordura adequadas & REGULAR ( ) IRREGULAR ( ) NSA ( ) \\
\hline Tubulações de saída e chegada & REGULAR ( ) IRREGULAR ( ) NSA ( ) \\
\hline Estado de conservação da tampa da fossa & REGULAR ( ) IRREGULAR ( ) NSA ( ) \\
\hline Estrutura da fossa & REGULAR ( ) IRREGULAR ( ) NSA ( ) \\
\hline Estanqueidade da fossa & REGULAR ( ) IRREGULAR ( ) NSA ( ) \\
\hline Estado de conservação do sumidouro & REGULAR ( ) IRREGULAR ( ) NSA ( ) \\
\hline Estrutura do sumidouro & REGULAR ( ) IRREGULAR ( ) NSA ( ) \\
\hline Estado de conservação da vala de infiltração & REGULAR ( ) IRREGULAR ( ) NSA ( ) \\
\hline Não conformidade no despejo de efluentes & REGULAR ( ) IRREGULAR ( ) NSA ( ) \\
\hline Escoamento em calha e ralos & REGULAR ( ) IRREGULAR ( ) NSA ( ) \\
\hline Estado de conservação das tubulações & REGULAR ( ) IRREGULAR ( ) NSA ( ) \\
\hline
\end{tabular}

Fonte: Autores 


\subsection{Criadouros}

Alguns empreendimentos têm como atividade a criação de animais, que demanda uma infraestrutura que favoreça o bem-estar dos animais durante a criação, funcionalidade e versatilidade na limpeza, conservação além de proporciona ao criador melhores índices de produtividade. As inspeções desses locais devem averiguar a conservação dos elementos estruturais, peças da cobertura, estanqueidade do local e as manifestações patológicas presentes conforme destacado no Quadro 4

Quadro 4 - Verificação de criadouros

\begin{tabular}{|c|l|}
\hline Descrição & \\
\hline Condição dos pilares estruturais & REGULAR ( ) IRREGULAR ( ) NSA ( ) \\
\hline Condição das vigas aparentes & $\operatorname{REGULAR~(~)~IRREGULAR~(~)~NSA~(~)~}$ \\
\hline Condição da laje de cobertura & $\operatorname{REGULAR~(~)~IRREGULAR~(~)~NSA~(~)~}$ \\
\hline Estado de conservação dos elementos estruturais & $\operatorname{REGULAR~(~)~IRREGULAR~(~)~NSA~(~)~}$ \\
\hline Peças da cobertura (estrutura e telhado) & $\operatorname{REGULAR~(~)~IRREGULAR~(~)~NSA~(~)~}$ \\
\hline Sistemas de climatização & $\operatorname{REGULAR~(~)~IRREGULAR~(~)~NSA~(~)~}$ \\
\hline Estanqueidade do local & $\operatorname{REGULAR~(~)~IRREGULAR~(~)~NSA~(~)~}$ \\
\hline
\end{tabular}

Fonte: Autores

\subsection{Postos de abastecimento}

Os postos de abastecimento armazenam líquidos inflamáveis que podem acarretar grandes riscos á saúde dos usuários, ao meio ambiente e toda comunidade em seu entorno. Segundo Menezes (2011), o vazamento destes tanques onde se armazenam os combustíveis pode provocar a contaminação dos solos e fontes de água, contribuindo para o risco eminente de explosões e incêndios. Por este motivo nesse tipo de edificação devemos avaliar com cautela o estado de preservação da edificação, para que evite qualquer exposição ao calor ou chamas, verificando também as condições das instalações elétricas de acordo com o Quadro 5 .

Quadro 5 - Verificação de postos de combustível

\begin{tabular}{|c|l|}
\hline Descrição & \\
\hline Coberturas & $\operatorname{REGULAR~(~)~IRREGULAR~(~)~NSA~(~)~}$ \\
\hline Presença de vazamentos & $\operatorname{REGULAR~(~)~IRREGULAR~(~)~NSA~(~)~}$ \\
\hline Odor característico do líquido inflamável & $\operatorname{REGULAR~(~)~IRREGULAR~(~)~NSA~(~)~}$ \\
\hline Sistema de combate a incêndio & $\operatorname{REGULAR~(~)~IRREGULAR~(~)~NSA~(~)~}$ \\
\hline Muretas de proteção & $\operatorname{REGULAR~(~)~IRREGULAR~(~)~NSA~(~)~}$ \\
\hline Disposição da fiação & $\operatorname{REGULAR~(~)~IRREGULAR~(~)~NSA~(~)~}$ \\
\hline Quadro de energia & $\operatorname{REGULAR~(~)~IRREGULAR~(~)~NSA~(~)~}$ \\
\hline Vedação & $\operatorname{REGULAR~(~)~IRREGULAR~(~)~NSA~(~)~}$ \\
\hline
\end{tabular}

Fonte: Autores

\section{Análise e classificação das irregularidades levantadas}

As inconformidades identificadas devem ser categorizadas em falhas ou anomalias considerando as causas da perda de desempenho da edificação. As anomalias são caracterizadas como endógenas ou construtivas, quando as manifestações patológicas decorrem das imprecisões nas etapas de projeto e/ou execuções, exógenas quando a perca do desempenho tem origem a partir de fatores degradantes externos e funcionais que derivam do envelhecimento natural e finalização da sua vida útil. As falhas são originadas do uso, operação e manutenções inadequadas (ABNT, 2020). 
Após a vistoria sensorial do imóvel e das instalações, a norma ABNT NBR 16747 (ABNT, 2020) orienta que sejam avaliadas e classificadasas irregularidades e manifestações patológicas levantadas, de acordo com os critérios de urgência, conforme evidenciado no Quadro 6. No laudo técnico deve constar as condições gerais do imóvel, orientações quantoas intervenções que devem ser efetuadas, considerando o grau de prioridade e risco eminente.

De acordo com Lanzinha (2002), a documentação elaborada pelo profissional habilitado deve apresentar linguagem coesa e de fácil compreensão para cliente.

Quadro 6 - Critérios de organização das prioridades em patamares de urgência

\begin{tabular}{|c|c|}
\hline Prioridade & Gravidade \\
\hline 1 & $\begin{array}{r}\text { Perda de desempenho compromete a saúde e/ou seguranças dos } \\
\text { usuários, do meio ambiente ou do próprio edifício }\end{array}$ \\
\hline 2 & $\begin{array}{r}\text { Perda parcial de desempenho que impacta a funcionalidade da } \\
\text { edificação, sem prejuízo à operação, sem comprometer a saúde } \\
\text { e/ou seguranças dos usuários }\end{array}$ \\
\hline 3 & Perda de desempenho pode ocasionar pequenos prejuízos à estética \\
\hline
\end{tabular}

\section{Conclusões}

Portanto a degradação de edificações rurais, de uso comercial e/ou habitacional, vem se mostrando frequente, devido intensos eventos climáticos, falta de orientação técnica e falhas na elaboração e execução do projeto.

O estudo da norma NBR 16747 (ABNT, 2020) torna-se relevante frente a padronização do processo de inspeção predial. A norma apresenta as diretrizes, conceitos básicos e metodologia que deve ser empregue para que ocorra uma avaliação precisa e de qualidade.

As etapas da inspeção apresentadas pela norma buscam auxiliar no diagnóstico assertivo das manifestações patológicas, orientando quanto a ações de uso e manutenibilidade, proporcionando a preservação da edificação e desempenho eficaz ao longo da sua vida útil. Os itens de verificação sugeridos neste estudo se mostraram satisfatórios para a finalidade proposta o que facilitou o processo de vistoria do empreendimento inspecionado.

Como proposta para futuras pesquisas recomenda-se inspecionar imóveis rurais de caráter habitacional, aplicando a metodologia apresentada pela NBR 16747 (ABNT, 2020), buscando levar informações técnicas quanto ao uso e manutenção das edificações às famílias que não têm ciência da importância da orientação técnica na construção civil ou não possuem recursos disponíveis para a contratação destes serviços.

\section{Agradecimentos}

Agradecemos ao Centro Federal de Educação Tecnológica de Minas Gerais - CEFET-MG pelo apoio direto. Agradecemos também ao LAR - Laboratório de Avaliação e Reabilitação de Ambiente Construído CEFET-MG - Campus Curvelo, pelo apoio durante o desenvolvimento do estudo. 


\section{Referências Bibliográficas}

Associação Brasileira De Normas Técnicas. NBR 14037: Diretrizes para elaboração de manuais de uso, operação e manutenção das edificações - Requisitos para elaboração e apresentação dos conteúdos. Rio de Janeiro, 2014.

Associação Brasileira De Normas Técnicas. NBR 16747: INSPEÇÃO PREDIAL - Diretrizes, Conceitos, Terminologias e Procedimentos. Rio de Janeiro, 2020.

Associação Brasileira De Normas Técnicas. NBR 15575-1: Edificações Habitacionais - Desempenho Parte 1: Requisitos gerais. Rio de Janeiro, 2013.

BRASIL. Ministério da Saúde. Gabinete do Ministro. Portaria no 2.914, de 12 de dezembro de 2011. Brasília, 2011.

Da Silva, A. G. Proposição de técnicas e modelos de gestão para o esgotamento sanitário em áreas rurais brasileiras. 2017.

Garrido, L. R.; Sehnem, S. Gestão associativa no empreendimento rural. Revista de Administração, v. 5, n. 8, p. 65-88, 2006.

Intituto Brasileiro de Avaliações e Perícia - IBAPE. Norma de Inspeção Predial Nacional. São Paulo, 2012.

Lanzinha, J. C.; Castro Gomes, J. P.; Peixoto Feitas, V. Application of a building inspection method to a group of rural houses. In: XXX IAHS World Congress on Housing. 2002. p. 9-13.

Menezes, A. M.; Moreira, C. A.; Ilha, L. M.; Schweig, C . Estudo geofísico de vazamento de combustíveis em posto de abastecimento. Geociências (São Paulo), v. 30, n. 4, p. 601-609, 2011.

Pujadas, F. Z. A. Inspeção Predial - Ferramenta de Avaliação da Manutenção. Disponível em: http://ibapenacional.com.br/biblioteca/wpcontent/uploads/2013/02/Inspecao-Predial-Ferramenta-de-AvaliacaodaManutencao.pdf. Acesso em 14 fev. 2021.

Sampaio, C. A. P., Terezo, R. F., Cardoso, C. O., Correggio, K. Colapsos De Edificações Rurais. Campo Grande 2014.

Sarmento, R. A.; Moraes, R. M., Viana, R. T. P.; Pessoa V. M; Carneiro, F. F. Determinantes socioambientais e saúde: O Brasil rural versus o Brasil urbano. Tempus Actas de Saúde Coletiva, v. 9, n. 2, p. ág. 221-235, 2015.

Verzola, S. N.; Marchiori, F. F.; Aragon, J. O. Proposta de lista de verificação para inspeção predial x urgência das manutenções. Encontro Nacional de Tecnologia do Ambiente Construído, XV ENTAC, Maceió: Alagoas (Brasil), p. 1226-1235, 2014. 